\title{
Pediatric Radiology: Why the Pediatricians Need it?
}

\author{
Arun Kumar Gupta ${ }^{1}$. Manohar Shroff ${ }^{2}$
}

Received: 18 March 2019 / Accepted: 18 March 2019 /Published online: 17 April 2019

(C) Dr. K C Chaudhuri Foundation 2019

Pediatric Medicine is no more a single specialty, rather it is a constellation of various subspecialties. With the expansion of each of these subspecialty, there is an increased need for improvement of the imaging modalities related to that specific area. Hence, keeping oneself updated with the newer developments in the field of Pediatric Radiology is the need of the hour for a pediatrician. The availability of a wide range of imaging modalities varying from radiograph to CT and MRI makes the choice of 'the right one' a really challenging task for the practitioners.

Keeping this urgent need of the pediatricians, Indian Journal of Pediatrics has decided to take out special symposium on 'Pediatric Radiology' in two issues. The first issue covers imaging in neonatal jaundice, bowel imaging (in two parts) and MRI of chest while the second issue will cover radiogenomics in inherited metabolic diseases, hydrocephalus in children, use of gadolinium based contrast in children and imaging in short stature.

Neonatal jaundice is a disease entity which haunts the physicians as well as the surgeons. Investigating a neonate with jaundice is a race against time, as early surgical intervention can significantly change the outcome. Differentiating surgical (especially extrahepatic biliary atresia) from non-surgical causes of jaundice is the most important question a radiologist has to answer. As Ultrasonography is the initial imaging modality, the radiologist should be well versed with the imaging findings of biliary atresia on ultrasonography. The roles of MRI and hepatobiliary nuclear scan should be well understood by the pediatrician as well as the pediatric surgeon; so as to avoid unnecessary delay in investigation and interpreta-

Arun Kumar Gupta

arunk676@gmail.com

1 Department of Radiodiagnosis, All India Institute of Medical Sciences, New Delhi 110029, India

2 Department of Diagnostic Imaging, Hospital for Sick Children/ Medical Imaging, University of Toronto, Toronto, Canada tion, and avoid any confusion. The first issue has an article by Pooja Abbey and her colleagues [1]. The authors have discussed causes of neonatal jaundice in great detail with supportive illustrations. Table 1 highlighting the features and criteria to diagnose biliary atresia on ultrasound should be very helpful since the first imaging investigation in all cases of neonatal jaundice will be an ultrasound. Further studies can be planned based on the possible diagnosis on ultrasound. Role of MRI and nuclear scan studies has been clearly elaborated.

Pediatric gastrointestinal imaging encompasses a wide range of disease entities enriched with complexities. The diagnostic conundrum in bowel pathology is often perplexing; leaving the physician in a dilemma about what modality to choose. The difficulties in bowel imaging include the convoluted morphology, and motility. However, newer fast imaging techniques in multidetector row CT scanners (MDCT); and faster MRI sequences have made this possible. In the recent issue, Devasenathipathy Kandasamy and colleagues have discussed the bowel pathologies in children in two sections [2,3]. Part 1 discusses the congenital and developmental disorders [2]; and part 2 deals with infective, inflammatory and neoplastic disorders [3]. Although it may sound too extensive; it is surprising to experience the lucidity with which the authors have described each entity. Esophageal atresia and tracheoesophageal fistula, duplication cyst, small bowel atresia and stenosis, meconium ileus, anorectal malformation are some of the commonly encountered developmental conditions encountered by both the pediatricians as well as the surgeons. Key imaging features of these entities should be known to all the pediatricians as well since many a times these patients may present during the emergency hours or first time in the OPD. Gastrointestinal tuberculosis is not uncommon in children in India. The incidence of Crohn's disease in childhood is also increasing. Differentiating these two disease entities is often very difficult and requires a very meticulous observation of imaging findings on CT/ MRI. In the part 2 of the bowel imaging article, the authors provides a comprehensive table with differentiating features of these two. The article also describes other clinically important entities such 
as necrotizing and neutropenic enterocolitis; and commonly occurring bowel tumors [3]. Overall, both the articles on bowel imaging have dealt with the whole spectrum of bowel diseases in children in depth with excellent illustrations which we are sure will be a very useful learning resource for reference to all the pediatricians.

For pathologies in pediatric chest, radiography and $\mathrm{CT}$ are usually the two most common imaging investigations done and are adequate in most cases for making the diagnosis. However MRI, with its many advantages especially in children due to its non-ionizing nature, also has very specific roles to play. The commonly known indications of thoracic MRI include mediastinal masses and lymphadenopathy, chest wall masses and tumors and pleural pathologies. It can act as a problem solving tool in pulmonary masses as well. In posterior mediastinal masses, MRI has an edge over CT in delineating the intraspinal extension with much conspicuity. Traditionally, MRI has been considered unsuitable for the lungs; as air in the lungs does not generate any MR signal. Also, respiratory artifacts and cardiac pulsation artifacts are major deterrants to good image quality in thoracic MRI, especially lung imaging in children. In the recent article on Pediatric Chest MRI, Kapur et al. have described the scope of thoracic MRI in children [4]. Newer fast and free breathing sequences in MRI have made lung MRI possible. Authors have repeatedly highlighted and compared role of CT vs. MRI in pediatric chest. In Table 2 they have gone in depth with this comparison for almost all the possible pathologies in pediatric chest. As MRI is the new tool in the imaging bandwagon, the practitioners must be well aware of the conditions in which they can be used. We are confident that this article will familiarize pediatricians not only with imaging features on MRI of various chest diseases in children but will also offer a rational approach to chest imaging especially the role of MRI.

This special symposium on 'Pediatric Radiology' by IJP, we believe, was very essential in order to update the pediatricians and pediatric surgeons on some of the important and common clinical problems which they encounter on a daily basis.

\section{Compliance with Ethical Standards}

Conflict of Interest None.

\section{References}

1. Abbey P, Kandasamy D, Naranje P. Neonatal jaundice. Indian J Pediatr. 2019. https://doi.org/10.1007/s12098-019-02856-0.

2. Kandasamy D, Sharma R, Gupta AK. Bowel imaging in children: part 1. Indian J Pediatr. 2019. https://doi.org/10.1007/s12098-01902877-9.

3. Kandasamy D, Sharma R, Gupta AK. Bowel imaging in children: part 2. Indian J Pediatr. 2019. https://doi.org/10.1007/s12098-01902878-8.

4. Kapur S, Bhalla AS, Jana M. Pediatric chest MRI: a review. Indian J Pediatr. 2019. https://doi.org/10.1007/s12098-018-02852-w.

Publisher's Note Springer Nature remains neutral with regard to jurisdictional claims in published maps and institutional affiliations. 